\title{
COVID-19 Pandemic in Bergamo outbreak: a call to war. Implementation of a "COVID-Hospital" model in ASST Bergamo-Est Trust as a disaster-response strategy.
}

\section{Edoardo laculli}

Department of Surgery, Seriate Hospital - ASST Bergamo Est, Bergamo, Italy

Michele Marini ( $\sim$ michele.marini@asst-bergamoest.it )

Azienda Socio Sanitaria Territoriale Bergamo Est https://orcid.org/0000-0003-1152-6756

Isabella Pezzoli

Universita degli Studi di Milano Facolta di Medicina e Chirurgia

Luisella Spinelli

Department of Surgery, Seriate Hospital - ASST Bergamo Est

\section{Lorenzo Vescovi}

Department of Surgery, Seriate Hospital - ASST Bergamo Est, Bergamo, Italy

\section{Silvia Frassini}

Department of Surgery, Seriate Hospital - ASST Bergamo Est, Bergamo, Italy

\section{Francesca Rubertà}

Department of Surgery, Seriate Hospital - ASST Bergamo Est, Bergamo, Italy

\section{Nadiane Paderno}

Department of Surgery, Seriate Hospital - ASST Bergamo Est, Bergamo, Italy

\section{Rosalba Della Nave}

Department of Surgery, Seriate Hospital - ASST Bergamo Est, Bergamo, Italy

\section{Elisa Da Re}

Universita degli Studi di Milano Facolta di Medicina e Chirurgia

\section{Hamade Abdel Meneme}

Department of Surgery, Seriate Hospital - ASST Bergamo Est, Bergamo, Italy

\section{Delia Beatrice Bonzi}

Department of Pharmacy, Alzano Hospital - ASST Bergamo Est, Bergamo, Italy

\section{Alberto Mario Guglielmo Leone Tedeschi}

Department of Medicine, Seriate Hospital - ASST Bergamo Est, Bergamo, Italy

\section{Alessandro laculli}

Department of Medical Oncology, Seriate Hospital - ASST Bergamo Est, Bergamo, Italy

\section{Giuseppe Nastasi}

Department of Medical Oncology, Seriate Hospital - ASST Bergamo Est, Bergamo, Italy 


\section{Pierpaolo Mariani}

Department of Surgery, Alzano Hospital - ASST Bergamo Est, Bergamo, Italy

\section{Antonio Piazzini Albani}

Department of Surgery, Seriate Hospital - ASST Bergamo Est, Bergamo, Italy

\section{Research article}

Keywords: Coronavirus, COVID-19, Pandemic, Mass Casualties Incident (MCI) response, Bergamo

Posted Date: June 22nd, 2020

DOI: https://doi.org/10.21203/rs.3.rs-36513/v1

License: (c) (1) This work is licensed under a Creative Commons Attribution 4.0 International License. Read Full License 


\section{Abstract}

Background: COVID-19 is a strikingly emerging disease caused by a new coronavirus (SARS-CoV-2) that has rapidly spread across all continents and affecting virtually every country. From a clinical, epidemiological, political and financial perspective, COVID-19 pandemic is now recognized as one of the worst disasters in modern era.

In the North of Italy, Bergamo has been the European equal to Chinese City of Wuhan in terms of epidemiological impact of COVID-19. The first case of SARS-Cov-2 infection in Bergamo has been reported in Alzano District General Hospital (DGH), part of ASST Bergamo-Est Multi Hospitals Network. ASST Bergamo-Est comprises four Hospitals serving more than $50 \%$ of Bergamo Province and with a patient catchment of 387000 inhabitants.

Methods: We retrospectively analyze the challenges and actual impact of the SARS-CoV-2 pandemic on the hospital capacity and performance. Accordingly, we report the specific operational procedures and clinical governance implementation related to the transformation of our Institutions into "COVIDhospitals".

Results: From 1st of March to 20th of April, 4919 consecutive patients were assessed in ASST BergamoEst Hospitals as "COVID-Hospital" referral centre, of these 1412 patients were admitted with diagnosis of moderate to severe COVID-19 respiratory insufficiency. Most of patients were high-risk individuals with a median age of 69 years. In-hospital mortality rate was $33.1 \%$. Specific performance improvements under the "COVID-19 Hospital model" capacity-expansion strategy were analysed.

Conclusions: This paper informs on the experience of ASST Bergamo-Est Trust and the disaster-response strategy during the COVID-19 pandemic. The ASST Bergamo-Est data reported corroborates the recent call for action to Governments for promoting MCI improved management with focus on COVID-19 pandemic and its possible recurrence.

\section{Background:}

COVID-19 is a strikingly emerging disease caused by a new coronavirus (SARS-CoV-2) that has rapidly spread across all continents and affecting virtually every country to such extent to force WHO (World Health Organization) to call for a pandemic alert on the 11th of March 2020. Considering the scale of the disease in terms of prevalence of infection, death toll and economic and social consequences, it is now universally recognized as the greatest disaster humanity has faced since the end of World War II.

The epidemiological burden of the disease has been so heavy that rapid exhaustion of local response resources and massive disruption to the delivery of care have been recorded invariably in every country. Many local hospitals in urban areas have been challenged by sudden surge of patients as experienced 
only in Level 5 and 6 Mass Casualty Incidents (MCls) and requiring statewide resources aid as per definition (> 1000 patients) (Table 1) [1].

Even highly performing and advanced National Health Systems in Europe and USA (United States of America) needed profound modifications to their service and activities to mitigate unprecedented loss of life.

Bergamo has been the first and worst affected city in Europe by SARS-CoV-2 infection, after its spread in Eastern Asian countries.

This paper informs on the experience of ASST Bergamo-Est Trust during the COVID-19 pandemic. The Trust is a Multi Hospitals Network (MHN) with a hub-and-spoke organization that includes Seriate and Alzano hub-hospitals and Piario and Lovere spoke-centres.

ASST Bergamo-Est has been among the first Medical centres in Italy to be appointed as "COVIDhospitals" in the context of the disaster-response strategy to face the $\mathrm{MCl}$ related to the outbreak of the disease in Italy. We analyze the challenges and actual impact of the pandemic on the hospital capacity and performance. Accordingly we report the specific operational procedures and clinical governance implementation related to the transformation of our Institutions into "COVID-hospitals".

\section{Methods:}

We describe the operational transformation as an emergency response during the unfolding of recent COVID-19 pandemic in ASST Bergamo-Est Trust Hospitals. Hospital capacity-expansion data and COVID19 mortality figures are reported.

All patients with possible COVID-like symptoms from Eastern Area of Bergamo Province were directly admitted or transferred from Residential Homes or neighbouring local hospitals to our Emergency Departments (ED) for appropriate triage and diagnostic confirmation and treatment.

"COVID-positive" patients were defined as those with COVID-like symptoms and direct or possible exposure to SARS-CoV-2 with either a radiological confirmation of interstitial pneumonia or positive SARS-CoV-2 PCR from nasopharyngeal swab. High-risk patients and patients triaged with moderate to severe symptoms were admitted.

From 1st of March to 20th of April, 4919 consecutive patients were assessed in ASST Bergamo-Est Hospitals ED Departments; of these, 1412 patients were admitted with diagnosis of moderate to severe COVID-19. The demographic data and clinical outcome have been retrospectively collected and investigated. Additional analysis was performed on the group of COVID-19 patients admitted and managed in the Surgical Department Units of the Trust.

The entrustment of large District General Hospitals (DGHs) as "COVID-Hospitals" model is part of a framework developed by Italian local and national authorities to manage the recent $\mathrm{MCl}$ related to 
Coronavirus infection in Lombardy.

On March the 1st, after appointment by Public Health Authorities as "COVID-Hospitals", a complete closure of elective and routine activities was completed and an institutional COVID-19 operational policy was implemented to revise the whole-trust capability and performance. All hospitals spaces, including wards and theatres, were revised for capacity-expansion strategy. Namely, surgical theatres and anaesthetic rooms were set-up and made available to be used as additional Intensive Treatment Units (ITUs). Since the beginning, Trust clinical staff in its entirety was re-allocated accordingly to help anaesthetists and physicians in the management of critically ill and respiratory compromised patients. Likewise, surgical teams were appointed to manage a Sub-Intensive Units with the support of appropriate in-Trust expertise.

Modifications of standard Clinical Governance in our Institutions, implementation of COVID-19 protocols within the Trust and operational policy coordination with public authorities are highlighted. Potentials for improving western hospitals preparedness for future similar events or $\mathrm{MCl}$, including possible SARS-CoV2 epidemic recurrence, are proposed.

COVID-19 management guidelines were prepared through multidisciplinary discussions amongst the relevant expertise within the Trust and made available to all clinical staff as standard of care.

Infection control measures across the hospital were targeted to minimize the risk of infection from the community and within the hospital. An enhanced Personal Protection Equipment (PPE) protocol for Health Care Workers in the Trust was established at the very early stage of COVID-19 Protocol, including FFP2/FFP3 masks and water-resistant full-body gown or bio-hazard suits, depending on the level of exposure to clinical areas.

\section{Results}

On 11th March WHO declared COVID-19 outbreak a pandemic, as numbers of cases outside China showed 13-fold increase and virus infection reached 113 countries with a death toll of 4291 lives [2].

On that date, Lombardy in Italy was the most affected region of the world outside China with a total of 12462 cases tested positive. The city of Bergamo was the worst outbreak in Europe with 2136 positive patients and an increase rate of 377 new cases per day [3].

By March the 1st and eight days after the Italian patient-zero of SARS-Cov-2 found in Lombardy on the 20th of February, ASST Bergamo-Est was identified by the Regional Government and entrusted to implement a strategic transitory re-qualification from large District General Hospital $\mathrm{MCH}$ to a dedicated “COVID-19 Hospital”.

From 1st of March to 20th of April we registered 4.919 Emergency Department referrals. Four hundred eighty-three patients (9,8\%) were transferred to other Hospitals; $2.768(56,3 \%)$ patients were discharged for self-isolation due to positive laboratory test to COVID-19; 1.412 (28,7\%) patients were admitted for 
medical treatment and respiratory support. Two hundred fifty-six (5.2\%) patient died in the Emergency Department. Among the 4,034 SARS-Cov-2 RT-PCR nasal-pharyngeal swabs performed, 2,357 (58,4\%) resulted positive. One thousand four hundred thirty-nine CT scan of the chest were performed. Four hundred and ninety $(19,6 \%)$ Health Care workers had a COVID-19 positive swab test; COVID-19 related mortality among Health Care Workers was $0,16 \%$ (Table 2).

Median age of admitted patients due to Moderate/Severe respiratory insufficiency is 69 , with $48.9 \%$ older than 70 and considered at high-risk. We recorded an in-hospital mortality rate of $33.1 \%$ (Table 2).

In the study period interval ASST Bergamo-Est implemented performance improvements under capacityexpansion strategy for Level $6 \mathrm{MCl}$. Increase in medical supplies, hospital expansion capacity and drugs and Oxygen consumption compared to the same period in 2019 are shown in Table 3.

\section{Discussion}

COVID-19 is now widely recognized as one of the greatest challenge to the humanity of this century from the clinical, epidemiological, political and financial perspective.

National Health Systems have been universally put under unprecedented strain by the scale of pandemic and the abrupt surge of population requiring medical attention and hospital admissions for critical care management within an abnormal short notice. Such an overwhelming discrepancy to meet the demand of health care is defined as Mass-Casualty Incident (MCl) by international emergency associations [1]. In modern era these events have become more frequent also in western societies (e.g. natural disasters, war scenarios, terrorist attacks) so to prompt experts to call for implementation of well coded strategies and guidelines to improve preparedness and coping ability of Health Systems in such scenarios [4-5].

When considering the SARS-Cov-2 pandemic figures, a massive disruption to Hospital-network at any level was to be expected, with unavoidable initial disarray never mind the level of infrastructure and resources. Such effect is also reported in many wealthy high-standard Health Systems (e.g. USA, UK, France, Italy).

As a matter of fact, the scale of COVID-19 disaster prompted several authors to advocate for a new $\mathrm{MCI}$ level definition of its own and beyond the Level VI for current pandemic and future similar casualties [4].

Namely, for the first time in modern era Western societies had to re-consider levels of care and public health priorities, so to quickly introduce deep modifications on an operational perspective and so to allow emergency response strategy to take over routine activities.

In this context, the Italian model encompasses the creation of so-called "COVID-Hospitals" from previously existing important District General Hospitals. This has proven to be a possible effective strategic response to mitigate the impact of the SARS-Cov-2 outbreak and relieve the burden of the pandemic across the National Health System. 
The Chinese City of Wuhan in Hubei Province has been internationally recognized as the first city affected by the SARS-Cov-2 outbreak that progressed into the COVID-19 pandemic (11 Million inhabitants, 50,333 COVID-19 confirmed cases; 457 cases/100,000 inhabitants; 3869 COVID-19 related deaths) [6]. From China and other Eastern Countries, the virus pandemic has then moved to western societies, with Italy being the first and worst-hit European country affected.

Bergamo Province, in the Italian northern District of Lombardy, has been the European equal to Wuhan for the COVID-19 pandemic in terms of epidemiological impact of SARS-Cov-2 infection: 1.1 million inhabitants; 10,788 COVID-19 confirmed cases; 980 cases/100,000 inhabitants; 2,835 COVID-19 related deaths [7].

Since the beginning of SARS-Cov-2 pandemic declaration by WHO on the 11th March, Bergamo has been the most severely affected city in Italy and in Europe. The rate of infection showed a peak of 399 cases per day in March. As of 25th of April -date of this article draft- the total number of positive tested cases is 10,689 with and considered the peak of epidemic curve in Italy (Fig .1) [8].

Similarly to Wuhan, the severity of the disaster overwhelming local Public Health called for extreme measures such as local hospitals closure, "lock-down" confinement measures for general population, emergency-state declaration, etc.

The first case of SARS-Cov-2 infection in Bergamo has been reported on the 23th of February in Alzano District General Hospital (DGH). The DGH is part of a Multi-Hospital Network (MHN) called ASST Bergamo-Est that comprises Alzano, Seriate, Piario and Lovere Hospitals and works as an organisational Unit within the Italian National Health System, serving more than $50 \%$ of Bergamo Province and with a patient catchment of 387000 inhabitants and a total number of 723 hospital beds on a routine basis.

By March the 1st and eleven days after the Italian patient zero of SARS-Cov-2 found in Lombardy on the 20th of February, ASST Bergamo-Est Trust was identified by the Regional Government and entrusted to implement a strategic transitory re-qualification from large District General Hospitals to a dedicated "COVID-19 Referral Centre", being amongst the first Italian "COVID-Hospital" Institutions in the front-line to face the Level $6 \mathrm{MCl}$ related to SARS-Cov-2 infection.

As a matter of fact the peculiarity of Multi Hospital Network (MHN) framework of the Trust has allowed a coordinated response between our Hospitals that served well within the institutional strategy for the COVID-19 disaster response (e.g. pooling benefits, procurement strategies, patients transfers between the centres, re-allocation of specialized services, optimization of intensive care expertise). The Clinical Governance of the Trust has been appropriately adjusted and implemented after being appointed as "COVID-Hospital". A structured framework strategy between the Public authorities and Local Health Services network has been enforced. Likewise, extreme measures have been taken to be part of a "bundled" damage-control response: full closure to clinical services, complete re-deployment of the clinical staff, hospital logistics modifications, maximum optimization of resources (Table 2). 
It is commonly stated that an hospital can better serve a larger number of critical patients if they arrive over a longer period than it can if fewer critical patients arrive all at once [9]. With this consideration, during COVID-19 epidemic burst in Lombardy, ASST Bergamo-Est Trusts had to face both larger number of critical patients arriving all at once for several weeks until the epidemic peak was reached (Fig. 1). During the interval period there were 4919 total ED referrals with 1412 patients admitted for COVIDrelated Moderate/Severe Respiratory Insufficiency requiring either critical care or ventilatory support, accounting for an average of 28 critically ill patients admitted each and single day for 7 weeks.

COVID-19 is known to have high in-hospital mortality rate for critically ill patients as documented in large series by several authors (mortality 50-61\%) [10-11]. Notably, we can demonstrate that pandemic mortality curve exhibits a different pattern than the conventional "tri-modal death distribution" described in other recent Level $5 \mathrm{MCls}$ (e.g. earthquake, flood, tsunami) (Fig. 2) [12]. To our knowledge, this is the first documented report in the modern literature that describes a specific pandemic mortality curve based on a large scale population-based cohort. As shown in our figures, mortality increase appears directly time depending (Fig. 3).

As of now, there is no study analysing hospital performance specifically related to $\mathrm{MCl}$ [13-14]. Using the mortality curve as a surrogate, we hypothesize that an initial steep rise of deaths in COVID-19 pandemic is related to the surge of patients requiring critical care and ventilatory support which rapidly overcomes the Hospitals resources. On that respect, we reported 256 patients (5.2\%) died in ED either with a "black code" (e.g. unavoidable death) or with severe respiratory insufficiency awaiting for transfer in ITU facilities not promptly available in the Trust or in neighbouring hospitals. As a matter of fact, the emergency response from a single Hospital can only mitigate and slow down the peak of mortality depending on the scale of the pandemic wave. Subsequently a significant improvement of mortality rate can only be achieved with a broader disaster-response strategy effectively co-ordinated on a Local and National level. ASST Bergamo-Est experience shows a similar drop of casualties after prompt multidimensional strategy implementation and including the re-qualification into "COVID-Hospital" (Fig. 3), which allowed an increase of service within days from the first documented COVID-tested patient.

Namely, a full hospital occupancy for COVID patients was achieved by closing all wards and routine activities, accounting for a total of 723 beds. A crucial $175 \%$ increase ITU/HDU bed availability was achieved; 968 new c-PAP helmets were purchased, ten-time the standard usage in the same interval. Accordingly, additional high 02-delivery systems set up in each ward to sustain such an increase in demand and a total of 333,245 liters of Oxygen were consumed across the Trust in the study period, accounting for a $908 \%$ increase of routine supply. A peak of 700 vials per day of rocuronium and cisatracurium were used for intubated patients (Table 3) (Fig. 4). An increase in clinical staff recruitment for direct patient care (including 39 new "ad-hoc" doctors) as well as ancillary services (decontamination service, laboratory and diagnostics) was granted. To comply with the Trust diagnostic protocol for COVID19, 1,439 chest CT scans were performed in the named period, 4034 SARS-CoV-2 PCR from nasopharyngeal swabs were carried out. 
National guidelines in USA dictate that Hospital should be self-sufficient for 48-96 hours in the aftermath of $\mathrm{MCl}$. The COVID outbreak has resembled a model of a "daily-MCl" for several weeks. As shown by our figures, between 1st of March and 21st April Bergamo Hospitals were in a constant "war-scenario" and facing $\mathrm{MCl}$ for a prolonged period of 7 weeks, if compared to international infamous "single-events" like the 2015 Paris attack ( 312 casualties with 129 reported deaths ) or the 2013 Boston Marathon bombing ( 264 casualties treated in 27 local hospitals with 3 reported deaths).

The ASST Bergamo-Est experience corroborates the recent call for action to Governments and Global Community recently promoted by Coccolini et al [4]. Improving and promoting $\mathrm{MCl}$ management with a particular focus on current COVID-19 pandemic and possible recurrence seems paramount and sensible. As shown from in our mortality curve, during a pandemic there is an interval of days before a significant surge of casualties; an improved $\mathrm{MCl}$ management preparedness would allow Authorities to implement planned response-strategies and potentially decrease the peak of mortality.

Since the early stage of the Pandemic unfolding, the implementation of a structured response framework in ASST Bergamo-Est has allowed to increase the service capacity compared to routine operation service to exceptional degree (Table 3). On the other hand, it is well-recognized by experts that the only way for a single Hospital to cope with high level $\mathrm{MCl}$ is a combination of focused and prompted modification of operational plan as well as inevitably lower the standards of care (e.g. acting outside the traditional duties as in surgeons managing sub-intensive units, reducing nurse to patients ratio, discontinuing routine activities, etc) (Table 2) [13-16]. Such a framework strategy can maximize services, allow effective capacity expansions so to mitigate the predictable peak of mortality and subsequently reducing it by avoiding indirect and avoidable loss of life. After such single Institution response, National Authorities intervention would help and co-ordinate neighbouring local Hospitals as well as a "nationbased" Health System response plan [17].

With no doubt, the first-line response to mass incidents and disasters would be a prompt and effective increase in performance by putting in place capacity-expansion strategies in every hospital involved.

With this respect, all Surgical and Medical Departments in ASST Bergamo-Est had to cancel non-essential operations and services and promptly discharge eligible patients. Although proven that clinical services closure is highly costly and disruptive, in such exceptional circumstance, it is considered crucial, in line with every international recommendation for Disaster Emergency Management [15-16]. Accordingly, the Local Government of Lombardy enacted a Regional Health Coordination task-force to reallocate oncologic and emergency patients from "COVID-Hospitals" in satellite Tertiary Referral Centres which kept specialist services running so to ensure continuity of care for all patients in need of surgical and specialist care.

As in other Infective Disease (ID) outbreaks and especially in COVID-19 pandemic, the disruption of Healthcare System has been particularly challenging due to the broadening discrepancy in terms of exponential surge of hospital care demand as opposed to relative deficiency of human clinical resources that is further worsened by Health Care Workers' (HCWs) nosocomial infection. 
Considering the exceptional transmissibility rate of SARS-Cov-2 novel virus, nosocomial transmission has been described universally. Data from literature report a HCW infection rate between 3 and 15\% [18]. In the setting of a "COVID-dedicated Hospital", care givers are at the very front line and highest risk of contagion and appropriate infection controls measures across the Trust and enhanced PPE-protocol for HCW are mandatory. Specifically HCWs infection rate between $3-15 \%$ in General Hospitals. Some authors report anecdotal evidence showing about $50 \%$ of ED staff tested positive, suggesting that a larger number of HCWs are actually being infected [19]. The actual rate of HCW is impossible to determine without comprehensive testing. Furthermore, in the context of pandemic like COVID-19 and in case of a positive HCW, it would be difficult to determine the actual source of infection from the hospital as opposed from the community. Italian Health Authorities report a percentage of $11.2 \%$ health professionals infected, with a mortality of $0.8 \%$ [20].

In our Institutions a total of $19.6 \% \mathrm{HCW}$ were tested positive and self-isolated accordingly. ASST Bergamo-Est have reported 4 COVID-19 related deaths $(0.16 \%)$ among the Trust staff.

The ASST Bergamo Est achieved low rate of Clinical Staff absence by enhancing the supply chain of PPE for HCWs during global Pandemic needs coordination with Local and National Authorities. In ASST Bergamo-Est "COVID-Hospital" model, daily availability of PPE for HCWs since the early days and without disruption was effectively guaranteed by implementation of Pharmacy Department supply (Table 3). Besides, Cleaning and Decontamination Services were also implemented in the Hospital public areas, the work-force shifts and sanitisation intervals were increased, specific COVID-disinfection protocol was implemented.

Considering the respiratory impairment of COVID-19 patients and the relatively limited numbers of ICU beds and Intensive Care Specialists, all Departments within ASST-Bergamo-Est were adjusted and set-up as "Sub-intensive Units" with adequate facilities and expertise to provide timely and appropriate Level-2 care to all COVID-19 patients with worsening respiratory function. This was particularly challenging in Alzano Hospital were no ITU facilities were routinely available before the pandemic. In this case, Surgical Team and Theatre Anaesthesists had to "team-work" and take the lead in managing patients with severe respiratory insufficiency patients that were intubated in theatres premises and awaiting appropriate transfer in due time.

In this article we have also focused on the organizational, clinical modifications and outcomes of ASSTBergamo Est Surgical Departments as an example of a Single Unit contribution within a "COVID-Hospital" strategy, highlighting the implementation of a dedicated Sub-intensive Care Units managed by surgical teams (Table 4) .

The total numbers of cPAPs and Mechanical Ventilators operating in the same time required oxygen supply implementation. In a single night work, an oxygen reservoir was set up and connected to the ward.

Around-the-clock support from Medical and Anaesthesiology Team were instituted to guarantee in-house training and skills acquisition for the management of clinical aspect of COVID-19 patients managed in 
the Surgical Unit.

National Guidelines on COVID-19 management (e.g. SIMIT - Italian Society for Infectious and Tropical Disease) were consulted to draft internal protocols which were progressively updated [20]. Considering the consent arising of new evidences from literature about COVID-19, medical staff was informed with weekly meetings and email bulletins.

As an example of a Surgical Unit implementation in the "COVID-19 Hospital" model, we revised our outcomes. From March the $1 \mathrm{~s}$ to April the 20th, 264 patients with COVID-19 related respiratory insufficiency were admitted to our Surgical Department: $73 \%$ had moderate/severe respiratory insufficiency at the admission, $51 \%$ of all patients needed c-PAP ventilatory support at some point of their management and $9 \%$ required intubation and transfer to ICU/HDU facilities [Tab 4]. We registered 32\% mortality rate which is comparable with the overall in-hospital mortality in ASST Bergamo-Est Trust (33\%) and more favourable than mortality reported in literature for critically ill COVID-19 patients [10-11]. On that note, a recent review of mortality rate related to other Level- $5 \mathrm{MCl}$ events shows similar outcomes.

Surgical expertise in triaging critical patients, multidisciplinary team-working, adaptability to work with limited resources and beyond traditional duties and ability to achieve good performance under stress circumstances served well in COVID-19 pandemic scenario, along with essential daily support by Intensivists and Physicians. With this preface, we advocate the direct and deep active involvement of surgical expertise in the Clinical Governance framework during severe $\mathrm{MCl}$, including pandemic disasters.

In light of our study focusing on ASST Bergamo-Est response to the recent $\mathrm{MCl}$ related to SARS-Cov-2 pandemic, as a medical community on the "front-line"we comment on the general narration of Hospitals' unpreparedness with the reported figures. An informed analysis of the COVID-19 outbreak at its peak would recognize the unavoidable rapid exhaustion of local resources during Level 5 and $6 \mathrm{MCl}$ scenarios (>1000 casualties) never mind the level of infrastructures. In Bergamo-Est Trust Hospitals there has been a total of 1412 admissions with an average of 28 patients/day requiring level of care $>2$ (ITU/HDU), each day for 7 weeks. In our study population, $65 \%$ of patient had severe respiratory insufficiency requiring high-level of care and advanced ventilatory support. A fair critical appraisal of MCls events and performance would acknowledge that no single Institution can plan to provide or made easily available an equivalent high number of ventilatory devices or ITU beds within few days, under any circumstances. In similar $\mathrm{MCl}$ scenarios, there is no such a thing as whole "preparedness" or "flawless" response and a "damage-control" strategy has to be considered [21].

\section{Conclusions}

We presented the data from Bergamo Province, as the worst affected area in Italy during the epidemiological peak of COVID-19.

We described the Italian example of ASST Bergamo-Est Trust re-qualification into "COVID-hospital" as a possible model of capacity-expansion strategy and operational modifications to cope with similar 
exceptional MCls, including possible SARS-Cov-2 future recurrence.

As demonstrated under such exceptional circumstances an initially abnormally high mortality rate relates to the scale of $\mathrm{MCl}$. A structured and prompt response at a Hospital-level can mitigate the disruption and avoid unnecessary further fatalities when associated to broader measures and strategic enforcements by Public Health Authorities.

\section{Abbreviations}

COVID-19

Coronavirus Disease 2019

SARS-CoV-2

Severe acute respiratory syndrome coronavirus 2

DGH

District General Hospital

ASST

Azienda Socio-Sanitaria Territoriale

$\mathrm{MCl}$

Mass Casualty Incident

WHO

World Health Organization

USA

United States of America

MHN

Multi Hospitals Network

ED

Emergency Departments

DGH

District General Hospitals

ITU

Intensive Treatment Unit

PPE

Enhanced Personal Protection Equipment

FFP

Filtering Face Piece

UK

United Kingdom

ID

Infective Disease

HCW

Health Care Workers 
SIMIT

Società Italiana di Malattie Infettive Tropicali

\section{Declarations}

\section{Ethics approval and consent to participate}

The study was performed in accordance with the Declaration of Helsinki. Informed consent from individual patients was waived because all data were anonymized and no sensible data were used for research purposes. According to ASST-Bergamo EST Trust policy, no ethical approval was needed.

\section{Consent for publication}

Not applicable

\section{Availability of data and materials}

The datasets used and/or analysed during the current study are available from the corresponding author on reasonable request.

\section{Competing interests}

All authors declare to have no competing interests.

\section{Funding}

Not applicable

\section{Authors' contributions}

El, MM Concept and design of the study

El, MM, IP Manuscript drafting

El, MM, IP Data collection and statistical analysis

LS, LV, SF, FR, NP, EDR, HAM, DBB, AT, Al, GN, PM, APA Critical Revision of the manuscript

El, APA Final approval of the draft

All authors have read and approved the manuscript.

\section{Acknowledgements}

Special thanks to Zaira Telini and Daniele Narno Ferri for their constant effort and precious help in collecting information and organizing database. 


\section{References}

1. Louisiana Emergency Response Network. Mass Casualty Incident (MCl) Level. http://lern.la.gov/lerndisaster-response/past-events/mass-casualty-incident-mci-levels/ (Accessed 25th of April 2020).

2. Ghebreyesus TA. World Health Organization. March 2020 https://www.who.int/dg/speeches/detail/who-director-general-s-opening-remarks-at-the-mediabriefing-on-covid-19-13-april-2020 (Accessed 25th of April 2020).

3. de. Italy: COVID-19 Cases (Coronavirus Disease) by regions and provinces. 2020. https://www.citypopulation.de/en/italy/covid/ (Accessed 25th of April 2020).

4. Coccolini, F., Sartelli, M., Kluger, Y. et al. COVID-19 the showdown for mass casualty preparedness and management: the Cassandra Syndrome. World J Emerg Surg 15, 26 (2020). https://doi.org/10.1186/s13017-020-00304-5

1. Ben-Ishay O, Mitaritonno M, Catena F, Sartelli M, Ansaloni L, Kluger Y. Mass casualty incidents-time to engage. World J Emerg Surg. 2016; 11:8.

6. National Health Commission of the Peoples Republic of China. April 20: Daily briefing on novel coronavirus cases in China. http://en.nhc.gov.cn/2020-04/20/c_79369.htm (Accessed 25th of April 2020).

7. com. Cumulative number of Coronavirus (COVID-19) cases in Italy since February 2020.

https://www.statista.com/statistics/1101680/coronavirus-cases-development-italy/ (Accessed 25th of April 2020).

1. Tariverdi M, Miller-Hooks E, Kirsch T. Strategies for Improved Hospital Response to Mass Casualty Incidents. Disaster Medicine and Public Health Preparedness. 2018;12(6):778-90.. 
1. Bhatraju PK, Ghassemieh BJ, Nichols M, Kim R, Jerome KR, Nalla AK, et al. Covid-19 in Critically III Patients in the Seattle Region - Case Series. New England Journal of Medicine. 2020;

1. Yang X, Yu Y, Xu J, Shu H, Xia J, Liu H, et al. Clinical course and outcomes of critically ill patients with SARS-CoV-2 pneumonia in Wuhan, China: a single-centered, retrospective, observational study. The Lancet Respiratory Medicine. 2020;8(5):475-81.

11. Smith, C. (Ed.). (2015). Trauma Anesthesia. Cambridge: Cambridge University Press. doi:10.1017/CBO9781139814713

1. Yi P, George SK, Paul JA, Lin L. Hospital capacity planning for disaster emergency management. Socio-Economic Planning Sciences. 2010;44(3):151-60.

1. Jacobson EU, Argon NT, Ziya S. Priority Assignment in Emergency Response. Operations Research. 2012;60(4):813-32.

14. World Health Organization. Mass casualty management systems. Strategies and guidelines for building health sector capacity. April 2007. https://www.who.int/hac/techguidance/tools/mcm_guidelinespdf

1. Hick JL, Barbera JA, Kelen GD. Refining Surge Capacity: Conventional, Contingency, and Crisis Capacity. Disaster Medicine and Public Health Preparedness. 2009;3(S1).

1. Center for Disease Control and Prevention Natural disasters and severe weather. https://www.cdc.gov/disasters/index.html Published 2016. (Accessed 15th of April 2020).

1. Cinar P, Kubal T, Freifeld A, Mishra A, Shulman L, Bachman J, et al. Safety at the Time of the COVID19 Pandemic: How to Keep Our Oncology Patients and Healthcare Workers Safe. Journal of the 
National Comprehensive Cancer Network. 2020;18(5):504-9.

1. Heneghan C, Oke J, Jefferson T COVID-19 How many Healthcare workers are infected?

Centre for Evidence Base Medicine . https://www.cebm.net/covid-19/covid-19-how-many-healthcareworkers-are-infected/ (Accessed 25th of April 2020).

1. Istituto Superiore di Sanita. Integrated surveillance of COVID-19 in Italy. https://www.epicentro.iss.it/coronavirus/bollettino/Infografica_28marzo\%20ENG.pdf (Accessed 25th of April 2020).

20. Vademecum per la cura delle persone con malattia da COVI-19. Versione 2.0, 13 marzo 2020 Available from: http://www.simit.org/medias/1568-covid19-vademecum-20-13-marzo-2020.pdf

21. Roberts DJ, Ball CG, Feliciano DV, Moore EE, Ivatury RR, Lucas CE, et al. History of the Innovation of Damage Control for Management of Trauma Patients: 1902-2016.

Annals of surgery. U.S. National Library of Medicine; 2017

Available from: https://www.ncbi.nlm.nih.gov/pubmed/27232248

\section{Tables}

Tab.1 Mass Casualty Incident Level definition (Louisiana Emergency Response Network - www.lern.la.gov).

Mass Casualty Incident (MCI) Levels

Level 1 5-10 potential victims

Level 2 10-20 potential victims

Level 3 20-100 potential victims

Level 4 100-1000 potential victims

Level 5 > 1000 victims

Level 6 Long-term operational period(s)/Pandemic 
Tab.2 ASST Bergamo Est Trust Clinical Governance and Operational Strategies after COVID-Hospital appointment

Page $17 / 22$ 
Hospital capacity-expansion strategy

Rapid surge of Hospital care in the population

Hospital clinical services closure

Patient allocation and transfer between Trust Hospitals

Cancelling non-essential operations

Early discharge of eligible patients

No pneumology service

24h Support Team Medical Emergency Team

Limited number of ICU beds

Expand ICU hospital capabilities (theatre suites, post-anesthesia rooms)

"Outreach Anaesthesist team" service across wards

Resource-constraints and service maximisation

Increased demand of radiology diagnostic service

New mobile CT scan purchase

Limited ventilatory devices

Purchase of c-PAP masks

Oxygen-supply implementation for every ward (O2 tank and supply connections set-up)

Clinical Staff shortage (sickness absence, self-isolation)

Daily PPE supply schedule

Sterility/Decontamination Hospital Staff Training

Newly graduated doctors enrolment

Staff-nurses recruitment.

“COVID - Trust” Guidelines

Implementation of COVID Management Protocol

Ongoing Audit and modification of current international evidences

Weekly audit and appraisal of Trust morbidity and mortality

Weekly trust e-mail bulletin and updates

In-house training by in-house expertise

c-PAP management

Antiviral therapy

International "COVID-Trial” with new drugs (eg Ruxolitinib)

Mixed/HDU Wards standard-of-care policy by Trust Clinical Committee

Multidisciplinary team-working

24h support team of Internal Medicine and Anesthesiologist

Daily Patients System Triage

- Green: transfer in dedicated low-intensity covid facilities

- Yellow: implementation support therapy (C-PAP, medical therapy, Tocilizumab, Steroids)

- Red: Intubation vs Palliative care

Daily contact and updates with patient families by responsible doctors

Regional Multi-Dimensional "COVID-Network"

ASST Bergamo-Est Multi Hospitals Network (Alzano - Seriate - Piario - Lovere)

GP's Community Medicine Response

Neighboring satellite Hospitals and Tertiary Referral Centres support

Private healthcare Systems involvement

Page $18 / 22$ 
Emergency Medical Services: ambulances and telephone help-line

Nursing-Homes networks

Local Public Health agencies (ASST Bergamo, Lombardy Regional Authority)

National Government law enforcement and funding

$\underline{\text { Tab.3 A and B. Hospital Performance and Service increase in ASST Bergamo-Est }}$

A. COVID-19 outbreak in ASST Bergamo-Est (1 ${ }^{\text {st }}$ March $-20^{\text {th }}$ April)

Emergency Department total referrals, N (\%)

4,919

ED mortality ( $<12$ hours in ED)

$256(5.2 \%)$

Transfer from ED

$483(9.8 \%)$

Admission

$1,412(28.7 \%)$

Discharge for self-isolation

$2,768(56.3 \%)$

In-hospital mortality, $\mathbf{N}(\%)$

$468(33.1 \%)$

Naso-pharyngeal Swabs, N (\%)

4,034

SARS-Cov-2 RT-PCR Positive

$2,357(58.4 \%)$

CT Thorax performed, $\mathrm{N}(\%)$

1,439

Health Care Workers COVID-19 positive, N (\%)

$490(19.6 \%)$

Swabbed in ED

$233(9.3 \%)$

Occupational Health Department indexed case

$257(10.3 \%)$

COVID-19 related HCW mortality

$4(0.16 \%)$

Admitted patients Demographics

Sex, N (\%)

M:F ratio

$789(56 \%)$ : $623(44 \%)$

Age (y), median (interval)

$69(54-80)$

$<50$ years, $\mathrm{N}(\%)$

$305(21,6 \%)$

50-70 years

$416(29,5 \%)$

$>70$ years

$690(48,9 \%)$

B. 
ASST Bergamo-Est Hospitals capacity expansion

ITU beds, $\mathbf{N}$

1. March-April 2020

$22(+175 \%)$

2. March-April 2019

8

C-PAP masks/helmets usage, $\mathbf{N}$

1. March-April 2020

$968(+1,014 \%)$

2. March-April 2019

79

Non-rebreather masks usage, $\mathrm{N}$

1. March-April 2020

$2,446(+1,123 \%)$

2. March-April 2019

200

Oxygen-supply usage $\left(\mathrm{m}^{3}\right), \mathrm{N}$

1. March-April 2020

$333,245(+908 \%)$

2. March-April 2019

33,051

Muscle-relaxant drugs usage (vials), $\mathrm{N}$

1. March-April 2020

$18,135(+885 \%)$

2. March-April 2019

1,840

Propofol drugs usage (grams), N

1. March-April 2020

$5,500(+442 \%)$

2. March-April 2019

1,014

Morphine drugs usage (vials), $\mathrm{N}$

1. March-April 2020

$6,050(+264 \%)$

2. March-April 2019

1,660

"Ad-hoc" COVID-19 Doctors recruitment, N 39

PPEs usage (items), $\mathrm{N}$

FFP2/FFP3

54,624

Surgical Mask

171,245

Water-tight Gowns / Bio-Hazard Suits44,321

\section{Table 4. Demographics and clinical outcome of patients admitted to Surgical COVID-19 Unit}

\begin{tabular}{|cl|}
\hline \multicolumn{2}{|c|}{ COVID- 19 in Surgical Wards $(\boldsymbol{n}=265)$} \\
\hline Sex, ratio & \\
M : F & $65 \%: 35 \%$ \\
Age, median (IQR) & $71(61-80)$ \\
< 50 yrs & $6 \%$ \\
$50-70$ yrs & $40 \%$ \\
$>70$ yrs & $54 \%$ \\
Acute Respiratory Failure, (\%) & \\
Mild & $23 \%$ \\
Moderate & $8 \%$ \\
Severe & $65 \%$ \\
Requiring c-PAP & $51 \%$ \\
Lenght of stay (days), mean (SD) & $12( \pm 9)$ \\
Discharged & $15( \pm 9)$ \\
Deceased & $8( \pm 5)$ \\
Outcome, (\%) & $8,8 \%$ \\
Transferred to ITU & $3 \%$ \\
Transferred to Step-Down Unit & $32.1 \%$ \\
In hospital mortality & \\
\hline
\end{tabular}

Page 20/22 


\section{Figures}

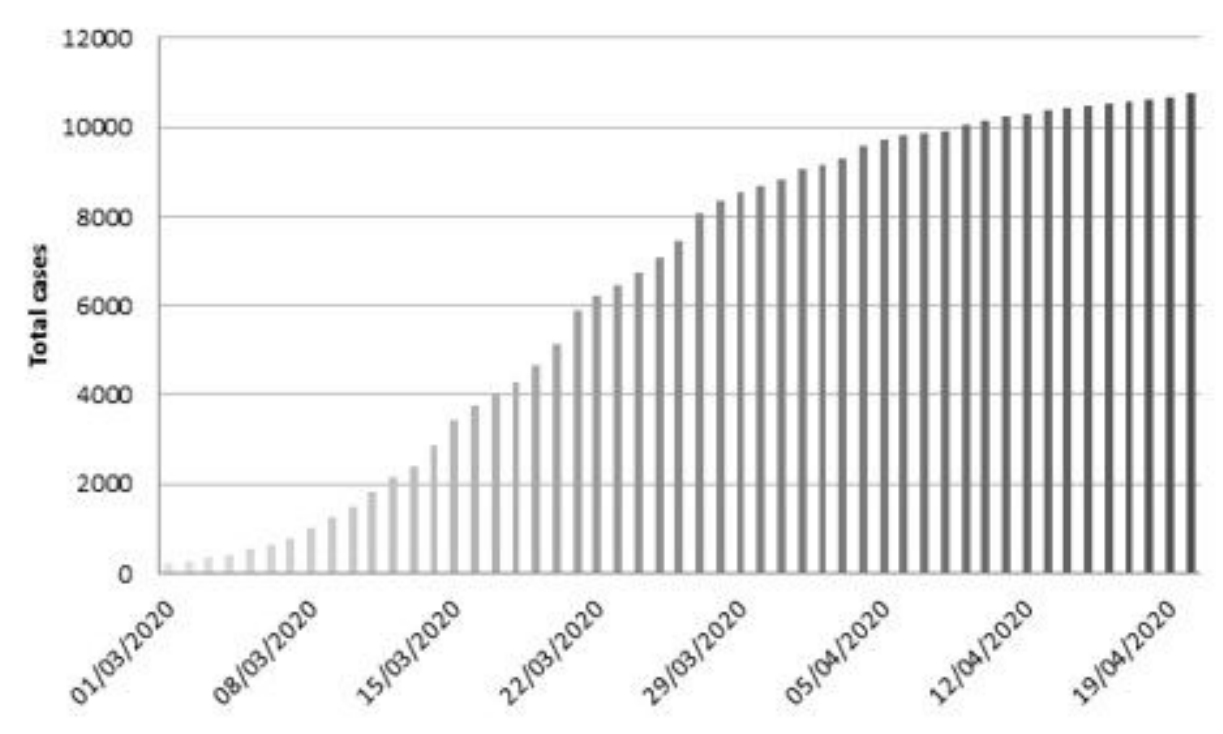

Figure 1

Epidemiological curve of SARS-Cov-2 infection in Bergamo province (1st March - 20th April 2020)

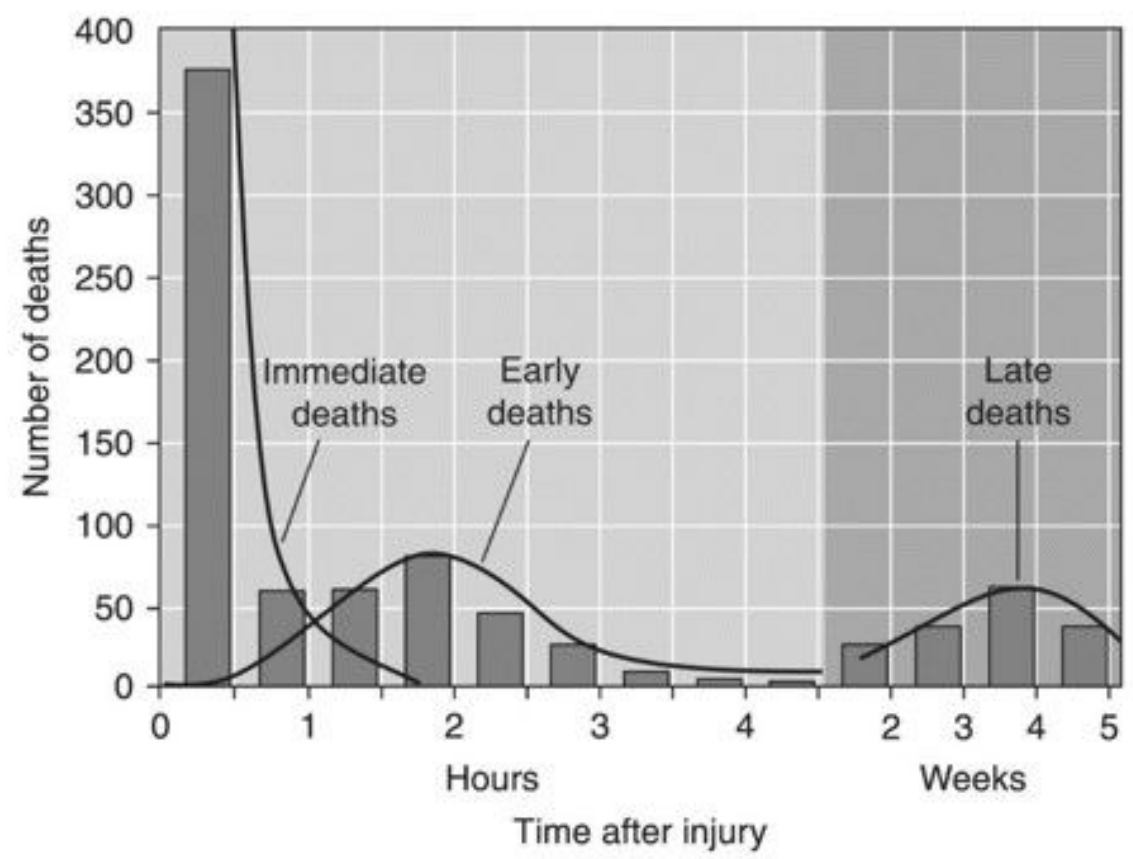

Page $21 / 22$ 
Figure 2

Tri-modal Death Distribution in $\mathrm{MCl}$

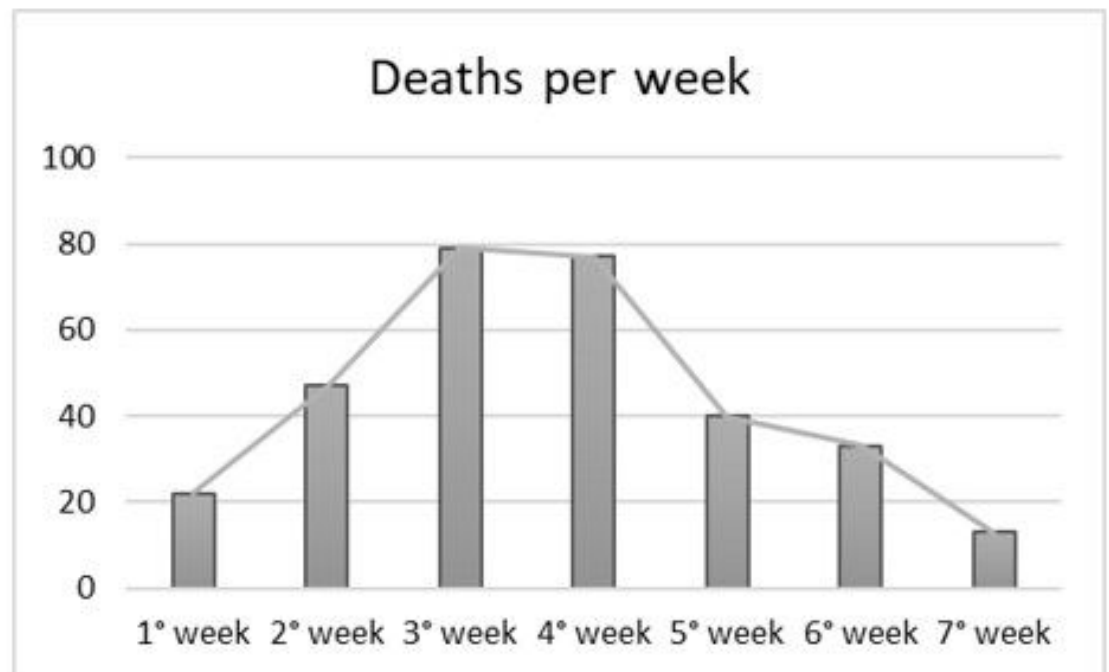

Figure 3

In-hospital mortality curve of COVID-19 Pandemic in ASST Bergamo-Est.

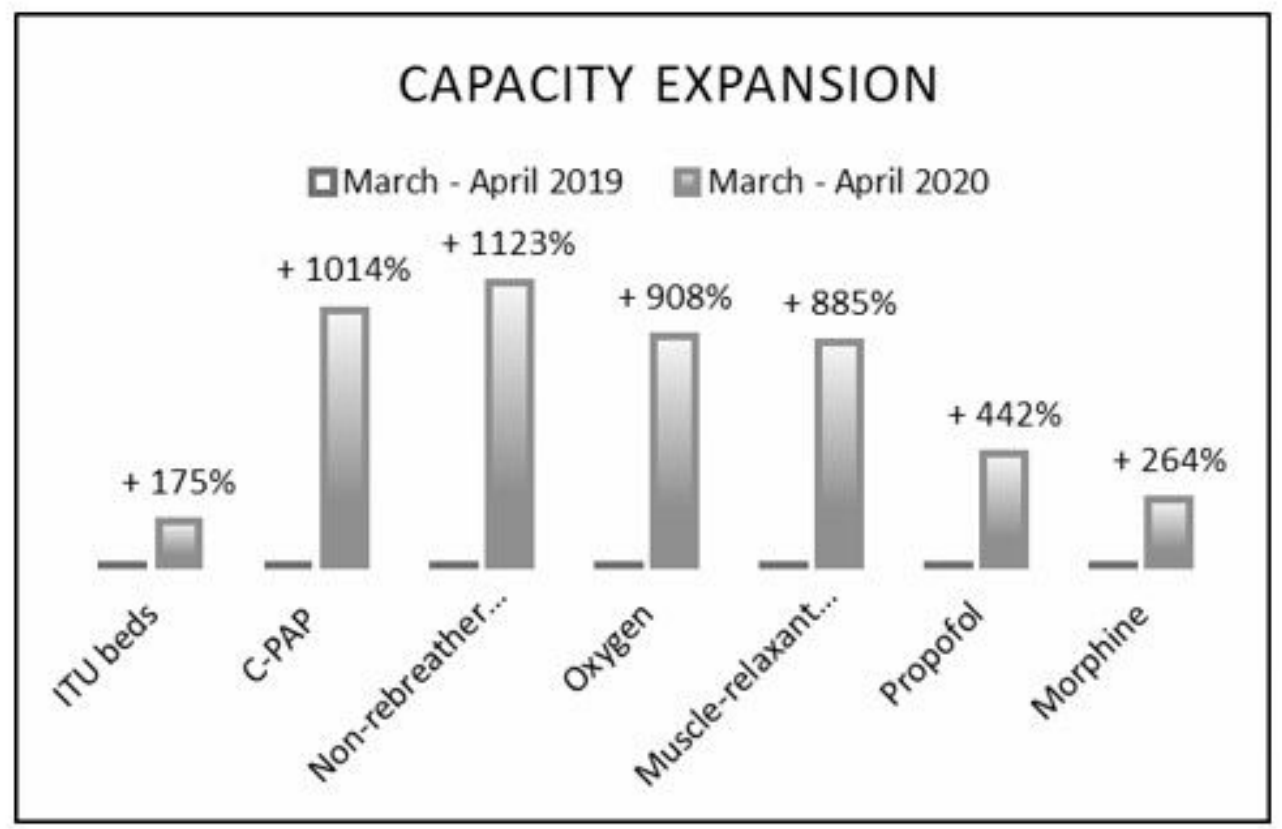

Figure 4

Hospital capacity-expansion outcome 